\title{
PATTERNS OF NON-RADIAL SOLUTIONS TO COUPLED SEMILINEAR ELLIPTIC SYSTEMS ON A DISC
}

\author{
ZALMAN BALANOV - EDWARD HOOTON — WIESLAW KRAWCEWICZ — DMITRII RACHINSKII
}

\begin{abstract}
In this paper, we prove the existence of non-radial solutions to the problem $-\triangle u=$ $\boldsymbol{f}(z, u),\left.u\right|_{\partial D}=0$ on the unit disc $D:=\{z \in \mathbb{C}:|z|<1\}$ with $u(z) \in \mathbb{R}^{k}$, where $\boldsymbol{f}$ is a sub-linear continuous function, differentiable with respect to $u$ at zero and satisfying $\boldsymbol{f}\left(e^{i \theta} z, u\right)=\boldsymbol{f}(z, u)$ for all $\theta \in \mathbb{R}, \boldsymbol{f}(z,-u)=-\boldsymbol{f}(z, u)$. Under the assumption that $\boldsymbol{f}$ respects additional (spacial) symmetries on $\mathbb{R}^{k}$, we investigate symmetric properties of the corresponding non-radial solutions. The abstract result is supported by a numerical example with extra $S_{4}$-symmetries.
\end{abstract}

\section{INTRODUCTION}

(a) Subject. It is a classical problem of mathematical physics to determine to which extend the solutions of elliptic BVPs inherit symmetric properties of the domain on which they are defined. For instance, the classical fixed membrane eigenvalue problem on a disc $D$, which is expressed as an eigenvalue Laplace problem with Dirichlet boundary conditions, admits a simple first eigenvalue with positive eigenfunction exhibiting full symmetries, while other eigenfunctions are nodal and demonstrate symmetry breaking. As it was established in [10, there exists a quite large class of similar nonlinear elliptic equations for which positive solutions exhibit full symmetries. For a detailed exposition of how symmetric properties of solutions (for nonlinear scalar Laplace equation) depend on geometric properties of associated energy functionals, we refer to the elegant survey by T. Weth [17.

In contrast to the scalar case, systems of elliptic BVPs lead to two different settings: variational and non-variational. In the first case, the existence of non-radial solutions was studied by many authors, see for example [3, 4, 5, 7, 14] and references therein. In the case of non-variational elliptic systems, there is a lot of interests in the existence of positive solutions. The standard method is based on establishing a priori bounds for positive solutions and using the so-called Liouville-type theorems and/or applying the Krasnoselskii's fixed-point theorem in a cone, see [8, 9, 15, 16] and the references therein.

In this paper we are interested in studying the existence of non-radial solutions to the following non-variational Laplace system on the $\operatorname{disc} D:=\{z \in \mathbb{C}:|z|<1\}$ :

$$
\left\{\begin{array}{l}
-\triangle u=f(z, u), \quad u(z) \in \mathbb{R}^{k}, \\
\left.u\right|_{\partial D}=0
\end{array}\right.
$$

\footnotetext{
2010 Mathematics Subject Classification. Primary: 35B06; Secondary: 47H11, 35J91.

Key words and phrases. Dirichlet Laplacian; non-radial solutions; equivariant Brouwer degree.

The first author Z. Balanov acknowledges the support from Xiangnan University. This work has been done as a part of the Prospective Human Resources Support Program of the Czech Academy of Sciences, and the second author E. Hooton acknowledges the support by this program. The third author W. Krawcewicz acknowledges the support from National Science Foundation of China through the grant no 11871171, and the support from Guangzhou University. The authors are also grateful to Thomas Bartsch and Jianshe Yu for their comments.
} 
where $\boldsymbol{f}: \bar{D} \times \mathbb{R}^{k} \rightarrow \mathbb{R}^{k}$ is a continuous odd radially symmetric function of sublinear growth, which is differentiable at zero. To be more precise, we assume that $\boldsymbol{f}$ satisfies the following conditions:

$\left(A_{1}\right) \boldsymbol{f}\left(e^{i \theta} z, u\right)=\boldsymbol{f}(z, u)$ for all $z \in D, u \in \mathbb{R}^{k}$ and $\theta \in \mathbb{R}$;

$\left(A_{2}\right) \boldsymbol{f}(z,-u)=-\boldsymbol{f}(z, u)$ for all $z \in D, u \in \mathbb{R}^{k}$;

$\left(A_{3}\right)$ there exists a $k \times k$-matrix $A, c>0$ and $\beta>1$ such that

$$
|\boldsymbol{f}(z, u)-A u| \leq c|u|^{\beta} \quad \text { for al } \quad z \in \bar{D}, u \in \mathbb{R}^{k}
$$

$\left(A_{4}\right)$ there exist $a, b>0$ and $\alpha \in(0,1)$ such that

$$
|\boldsymbol{f}(z, u)|<a|u|^{\alpha}+b \quad \text { for al } \quad z \in \bar{D}, u \in \mathbb{R}^{k} .
$$

Observe that conditions $\left(A_{1}\right)$ and $\left(A_{2}\right)$ imply that system (11) is $G:=O(2) \times \mathbb{Z}_{2}$-symmetric.

Observe that given an orthogonal $G$-representation $V$ (here $G$ stands for a compact Lie group) and an admissible $G$-pair $(f, \Omega)$ in $V$ (i.e., $\Omega \subset V$ is an open bounded $G$-invariant set and $f: V \rightarrow V$ a $G$-equivariant map without zeros on $\partial \Omega)$, the Brouwer degree $d_{H}:=\operatorname{deg}\left(f^{H}, \Omega^{H}\right)$ is well-defined for any $H \leq G$ (here $\Omega^{H}:=\{x \in \Omega: h x=x \forall h \in H\}$ and $f^{H}:=\left.f\right|_{\Omega^{H}}$ ). Then, if for some $H$, one has $d_{H} \neq 0$, the existence of solutions with symmetry at least $H$ to equation $f(x)=0$ in $\Omega$, can be predicted. Although this approach provides a way to determine the existence of solutions in $\Omega$, and even to distinguish their different orbit types, nevertheless, it comes at a price of elaborate $H$-fixed-point space computations which can be a rather challenging task.

(b) Method. Our method is based on the usage of the Brouwer equivariant degree theory (cf. [2, 1]; see also [3]). To be more explicit, the equivariant degree $G-\operatorname{deg}(f, \Omega)$ is an element of the free $\mathbb{Z}$-module $A_{0}(G)$ generated by the conjugacy classes $(H)$ of subgroups $H$ of $G$ with a finite Weyl group $W(H)$ :

$$
G-\operatorname{deg}(f, \Omega)=\sum_{(H)} n_{H}(H), \quad n_{H} \in \mathbb{Z},
$$

where the coefficients $n_{H}$ are given by the following recurrence formula

$$
n_{H}=\frac{d_{H}-\sum_{(L)>(H)} n_{L} n(H, L)|W(L)|}{|W(H)|},
$$

and $n(H, L)$ denotes the number of subgroups $L^{\prime}$ in $(L)$ such that $H \leq L^{\prime}$ (see [2]). Also, we use the notation

$$
\operatorname{coeff}^{H}(a):=n_{H} \text { for any } a=\sum_{(H)} n_{H}(H) \in A_{0}(G) .
$$

One can immediately recognize a connection between the two collections: $\left\{d_{H}\right\}$ and $\left\{n_{H}\right\}$, where $H \leq G$ and $W(H)$ is finite. As a matter of fact, $G-\operatorname{deg}(f, \Omega)$ satisfies the standard properties expected from any topological degree. However, there is one additional functorial property, which plays a crucial role in computations, namely the multiplicativity property. In fact, $A_{0}(G)$ has a natural structure of a ring (which is called the Burnside ring of $G$ ), where the multiplication $\cdot: A_{0}(G) \times A_{0}(G) \rightarrow A_{0}(G)$ is defined on generators by $(H) \cdot(K)=\sum_{(L)} m_{L}(L)$ with

$$
m_{L}:=\left|(G / H \times G / K)_{(L)} / G\right|, \quad \text { where } W(L) \text { is finite. }
$$

The multiplicativity property for two admissible $G$-pairs $\left(f_{1}, \Omega_{1}\right)$ and $\left(f_{2}, \Omega_{2}\right)$ means the following equality

$$
G-\operatorname{deg}\left(f_{1} \times f_{2}, \Omega_{1} \times \Omega_{2}\right)=G-\operatorname{deg}\left(f_{1}, \Omega_{1}\right) \cdot G-\operatorname{deg}\left(f_{2}, \Omega_{2}\right) .
$$

Given a $G$-equivariant linear isomorphism $A: V \rightarrow V$, formula (8) combined with the equivariant spectral decomposition of $A$, reduces the computations of $G$-deg $(A, B(V))$ to the computation of the so-called basic degrees $\operatorname{deg}_{\mathcal{V}_{i}}$, which can be 'prefabricated' in advance for any group $G$ (here 
$\operatorname{deg}_{\mathcal{V}_{i}}:=G-\operatorname{deg}\left(-\operatorname{Id}, B\left(\mathcal{V}_{i}\right)\right)$ with $\mathcal{V}_{i}$ being an irreducible $G$-representation and $B(X)$ stands for the unit ball in $X)$.

With an eye towards making our presentation acceptable for non-experts in the equivariant degree theory, we first obtain the existence result assuming that system (1) respects $O(2) \times \mathbb{Z}_{2^{-}}$ symmetries only. To show the full power of the proposed method, we next consider system (11) in the presence of extra spacial symmetries assuming that $\mathbb{R}^{k}$ is equipped with the structure of $\Gamma$ representation respected by $\boldsymbol{f}$, in which case, we establish minimal symmetries of the corresponding non-radial solutions. The obtained abstract result is supported by a numerical example with extra $\Gamma=S_{4}$-symmetries. In this case, the computations of the related Brouwer $O(2) \times \Gamma \times \mathbb{Z}_{2}$-equivariant degrees can be assisted by GAP computer programs (the GAP code is listed in Appendix B). Observe also that the method can be easily adopted to treat other symmetric domains.

\section{Functional Spaces Reformulation and a priori Bounds}

Consider the Sobolev space $\mathscr{H}:=H_{0}^{2}\left(D, \mathbb{R}^{k}\right)$ equipped with the usual norm $\|u\|:=\max \left\{\left\|D^{s} u\right\|_{L^{2}}\right.$ : $|s| \leq 2\}$, where $s=\left(s_{1}, s_{2}\right),|s|=s_{1}+s_{2}$, and $D^{s} u=\frac{\partial^{|s|}}{\partial^{s_{1}} x \partial^{s_{2} y}}$. Define the linear operator $\mathscr{L}: \mathscr{H} \rightarrow L^{2}\left(D ; \mathbb{R}^{k}\right)$ by

$$
\mathscr{L} u:=-\triangle u, \quad u \in \mathscr{H} .
$$

It is well known that $\mathscr{L}$ is an isomorphism, i.e., the inverse operator $\mathscr{L}^{-1}$ is well defined and bounded.

Choose

$$
q>\max \{1,2 \alpha\}
$$

(for example, it is enough to take $q:=2 \beta$, cf. assumptions (A3) and (A4)). Denote by $j: \mathscr{H} \rightarrow$ $L^{q}\left(D ; \mathbb{R}^{k}\right)$ the standard Sobolev embedding. Notice that under the assumption $\left(A_{4}\right)$, the function

$$
N(v)(z):=\boldsymbol{f}(z, v(z)), \quad z \in \bar{D}
$$

belongs to $L^{2}\left(D, \mathbb{R}^{k}\right)$ for any $v \in L^{q}\left(D ; \mathbb{R}^{k}\right)$. Indeed, consider $v \in L^{q}\left(D ; \mathbb{R}^{k}\right)$. Then, combining (9) and $\left(A_{4}\right)$ with the Hölder inequality, one has:

$$
\begin{aligned}
\|N(v)\|_{L^{2}} & \leq a\left\||v|^{\alpha}\right\|_{L^{2}}+b \sqrt{\pi} \\
& =a\left(\int_{D}|v|^{2 \alpha}\right)^{\frac{1}{2}}+b \sqrt{\pi} \\
& \leq a \pi^{\frac{1-2 \alpha / q}{2}}\left(\int_{D}|v|^{\frac{2 \alpha q}{2 \alpha}}\right)^{\frac{2 \alpha}{2} q}+b \sqrt{\pi} \\
& =a \pi^{1 / 2-\alpha / q}\|v\|_{L^{q}}^{\alpha}+b \sqrt{\pi} .
\end{aligned}
$$

Notice that system (11) is equivalent to the equation

$$
\mathscr{L} u=N(j u), \quad u \in \mathscr{H},
$$

which can also be written as

$$
\mathscr{F}(u):=u-\mathscr{L}^{-1} N(j u)=0, \quad u \in \mathscr{H},
$$

with a well defined nonlinear operator $\mathscr{F}: \mathscr{H} \rightarrow \mathscr{H}$.

We will need the following lemmas.

Lemma 2.1. Let $\boldsymbol{f}: \bar{D} \times \mathbb{R}^{k} \rightarrow \mathbb{R}^{k}$ be a continuous function satisfying the assumption $\left(A_{4}\right)$, then there exists a constant $R>0$ such that $\|u\|_{\mathscr{H}}<R$ for any solution $u \in \mathscr{H}$ to system (1). 
Proof. Assume that $u \in \mathscr{H}$ is a solution to (1), which implies that $u=\mathscr{L}^{-1} N(j u)$. Combining this with $\|N(j u)\|_{L^{2}} \leq a \pi^{(1-\alpha) / 2}\|u\|_{L^{2}}^{\alpha}+b \sqrt{\pi}$ (cf. $\left(A_{4}\right)$ implies

$$
\|u\|_{\mathscr{H}} \leq a \pi^{(1-\alpha) / 2}\left\|\mathscr{L}^{-1}\right\|_{L^{2} \rightarrow \mathscr{H}}\|u\|_{L_{2}}^{\alpha}+b \sqrt{\pi}\left\|\mathscr{L}^{-1}\right\|_{L^{2} \rightarrow \mathscr{H}} .
$$

Combining (14) with $\|u\|_{\mathscr{C}} \geq\|u\|_{L^{2}}$ yields

$$
\|u\|_{L^{2}} \leq c\|u\|_{L^{2}}^{\alpha}+d
$$

where $c:=a \pi^{(1-\alpha) / 2}\left\|\mathscr{L}^{-1}\right\|_{L^{2} \rightarrow \mathscr{H}}, d:=b \sqrt{\pi}\left\|\mathscr{L}^{-1}\right\|_{L^{2} \rightarrow \mathscr{H}}$. Then, since $0<\alpha<1$, there exists $R_{o}>0$ such that $\psi(t):=t-c t^{\alpha}-d>0$ for $t \geq R_{o}$. Consequently, $\|u\|_{L^{2}}<R_{o}$, and by (14),

$$
\|u\|_{\mathscr{H}} \leq c\|u\|_{L^{2}}^{\alpha}+d<c R_{o}^{\alpha}+d=: R
$$

is the required constant.

We also define the linear operator $\mathscr{A}: \mathscr{H} \rightarrow \mathscr{H}$, by

$$
\mathscr{A}(u)(z):=u-\mathscr{L}^{-1} A u(z), \quad u \in \mathscr{H}, z \in \bar{D} .
$$

Lemma 2.2. Under the assumptions $\left(A_{3}\right)$ and $\left(A_{4}\right)$, the nonlinear operator $\mathscr{F}: \mathscr{H} \rightarrow \mathscr{H}$ given by (13) is a completely continuous field differentiable at $0 \in \mathscr{H}$ with $D \mathscr{F}(0)=\mathscr{A}$.

Proof. Take $q:=2 \beta$ (cf. conditions $\left(A_{3}\right),\left(A_{4}\right)$ and (91)). Then, it follows from (11) that the operator $N$ given by (10) takes $L^{q}\left(D ; \mathbb{R}^{k}\right)$ to $L^{2}\left(D ; \mathbb{R}^{k}\right)$. Combining this with continuity of $\boldsymbol{f}$ and the classical Krasnoselskii's theorem (see [12, 13]), one obtains that the operator $N$ is continuous. Also, by the Rellich-Kondrachov Theorem (see, for instance, 6], Theorem 9.16 and Remark 20, p. $290), H_{0}^{1}(D ; \mathbb{R})$ is compactly embedded into $L^{q}(D ; \mathbb{R})$. This together with continuous embedding of $H_{0}^{2}(D ; \mathbb{R})$ into $H_{0}^{1}(D ; \mathbb{R})$ implies a compact embedding of $\mathscr{H}$ into $L^{q}\left(D ; \mathbb{R}^{2}\right)$. Thus, $\mathscr{F}$ is a completely continuous vector field.

Let us show that $N: L^{q}\left(D ; \mathbb{R}^{2}\right) \rightarrow L^{2}\left(D ; \mathbb{R}^{2}\right)$ is differentiable at the origin and the derivative coincides with the operator generated by $A$. In fact,

$$
\begin{aligned}
\frac{1}{\|u\|_{L^{q}}^{2}} \int_{D}|\boldsymbol{f}(z, u(z))-A u(z)|^{2} d z & \leq \frac{c^{2}}{\|u\|_{L^{q}}^{2}} \int_{D}|u(z)|^{2 \beta} d z \\
& =\frac{c^{2}}{\|u\|_{L^{q}}^{2}}\|u\|_{L^{q}}^{q}=c^{2}\|u\|_{L^{q}}^{2(\beta-1)},
\end{aligned}
$$

which implies that

$$
\lim _{\|u\|_{L^{q} \rightarrow 0}} \frac{\|N u-A u\|_{L^{2}}}{\|u\|_{L^{q}}} \leq c^{2} \lim _{\|u\|_{L^{q} \rightarrow 0}}\|u\|_{L^{q}}^{\beta-1}=0 .
$$

Combining this with boundedness of the linear operators $j$ and $\mathscr{L}^{-1}$ completes the proof of the lemma.

\section{Existence Results}

By passing to polar coordinates, one can easily compute the spectrum of the operator $\mathscr{L}$ (considered as an unbounded operator in $L^{2}\left(D ; \mathbb{R}^{k}\right)$. Namely, denote by $s_{n m}$ the $n$-th positive zero of the Bessel function $J_{m}$ of the first kind. Define

$$
\sigma(\mathscr{L})=\left\{s_{n m}: n \in \mathbb{N}, m=0,1,2, \ldots\right\} .
$$

Then, for each eigenvalue $s_{n m}$, the corresponding eigenspace can be described as follows (here we use the standard polar coordinates $(r, \theta))$ :

$$
\mathscr{E}_{n m}:=\left\{J_{m}\left(s_{n m} r\right)(\cos (m \theta) \vec{a}+\sin (m \theta) \vec{b}): \vec{a}, \vec{b} \in \mathbb{R}^{k}\right\}
$$


Put

$$
\mathscr{H}_{m}:=\overline{\bigoplus_{n=1}^{\infty} \mathscr{E}_{n m}} \quad \text { and } \quad \mathscr{A}_{m}:=\left.\mathscr{A}\right|_{\mathscr{E}_{m}}
$$

where the closure is taken in $\mathscr{H}$. It is clear that $\mathscr{A}\left(\mathscr{E}_{n m}\right) \subset \mathscr{E}_{n m}$, so $\mathscr{A}_{n m}:=\mathscr{A}_{\mathscr{E}_{n m}}: \mathscr{E}_{n m} \rightarrow \mathscr{E}_{n m}$ and consequently $\mathscr{A}_{m}: \mathscr{H}_{m} \rightarrow \mathscr{H}_{m}$.

Let us denote by $\sigma(A)$ the real spectrum of the matrix $A$ and by $\sigma_{+}(A)$ its positive spectrum. We make an additional "non-degeneracy" assumption:

(D) For all $\mu \in \sigma(A)$ and $n \in \mathbb{N}$ and $m=0,1,2, \ldots$, we have $s_{n m} \neq \mu$.

The assumption (D) implies that $\mathscr{A}$ is an isomorphism. Indeed, the real spectrum $\sigma(\mathscr{A})$ can be easily described:

$$
\sigma(\mathscr{A})=\bigcup_{m=0}^{\infty} \sigma\left(\mathscr{A}_{m}\right) \quad \text { where } \sigma\left(\mathscr{A}_{m}\right)=\left\{1-\frac{\mu}{s_{n m}}: \mu \in \sigma(A), n \in \mathbb{N}\right\} .
$$

For every $\mu \in \sigma_{+}(A)$ and $m=0,1,2,3, \ldots$, put

$$
\mathfrak{n}_{m}(\mu):=\left|\left\{(n, m): n \in \mathbb{N}, s_{n m}<\mu\right\}\right|,
$$

where $|X|$ stands for the cardinality of the set $X$. As is well known (see, for example, [18, p. 486), $s_{1 m}>\sqrt{m(m+2)}$, from which it follows that the numbers $\mathfrak{n}_{m}$ are non-zero only for finitely many $m=1,2,3 \ldots$ We also put

$$
\mathfrak{m}_{m}:=\sum_{\mu \in \sigma_{+}(A)} \mathfrak{n}_{m}(\mu) \cdot m(\mu)
$$

where $m(\mu)$ stands for the algebraic multiplicity of the eigenvalue $\mu$.

Now we can formulate our main existence result.

Theorem 3.1. Under the assumptions $\left(A_{1}\right)\left(A_{4}\right)$ and $(D)$, assume that there exists $m>0$ such that $\mathfrak{m}_{m}$ is odd. Then, system (11) admits a non-radial solution.

Proof. Notice that the group $G:=O(2) \times \mathbb{Z}_{2}$ acts naturally on the space $\mathscr{H}$ :

$$
(h, \pm 1)(u)(z):= \pm u\left(h^{-1} z\right), \quad\left(h \in O(2), \mathbb{Z}_{2}=\{ \pm 1\}, z \in D\right),
$$

and the nonlinear operator $\mathscr{F}$ is $G$-equivariant. By assumption (D), the linear operator $\mathscr{A}: \mathscr{H} \rightarrow$ $\mathscr{H}$ is an isomorphism, and by Lemma 2.2. there exists an $\varepsilon>0$ such that $\mathscr{F}$ is $B_{\varepsilon}(0)$-admissibly $G$-equivariantly homotopic to $\mathscr{A}$ (here $B_{\varepsilon}(0)$ stands for the disc in $\mathscr{H}$ of radius $\varepsilon$ centered at the origin). Similarly, by Lemma 2.1 the nonlinear operator $\mathscr{F}$ is $B_{R}(0)$-admissibly $G$-equivariantly homotopic to Id. Put, $\Omega:=B_{R}(0) \backslash \overline{B_{\varepsilon}(0)}$. Then, by the additivity property of the Brouwer $G$-equivariant degree, we have

$$
\begin{aligned}
G-\operatorname{deg}(\mathscr{F}, \Omega) & =G-\operatorname{deg}\left(\mathscr{F}, B_{R}(0)\right)-G-\operatorname{deg}\left(\mathscr{F}, B_{\varepsilon}(0)\right) \\
& =G-\operatorname{deg}\left(\operatorname{Id}, B_{R}(0)\right)-G-\operatorname{deg}\left(\mathscr{A}, B_{\varepsilon}(0)\right)=(G)-G-\operatorname{deg}\left(\mathscr{A}, B_{\varepsilon}(0)\right) \\
& =(G)-\prod_{m=0}^{\infty} G-\operatorname{deg}\left(\mathscr{A}_{m}, B\left(\mathscr{H}_{m}\right)\right) \\
& =(G)-G-\operatorname{deg}\left(\mathscr{A}_{0}, B\left(\mathscr{E}_{0}\right)\right) \cdot \prod_{m=1}^{\infty}\left(\operatorname{deg}_{\mathcal{V}_{m}^{-}}\right)^{\mathfrak{m}_{m}},
\end{aligned}
$$

where $\operatorname{deg}_{\mathcal{V}_{m}^{-}}:=G$-deg $\left(-\mathrm{Id}, B\left(\mathcal{V}_{m}^{-}\right)\right)$and $\mathcal{V}_{m}^{-}$stands for the $m$-th irreducible $G$-representation. Notice that $G-\operatorname{deg}\left(\mathscr{A}_{0}, B\left(\mathscr{E}_{0}\right)\right)$ is equal either to $(G)$ or to $(G)-(O(2))$ and $\operatorname{deg}_{\mathcal{V}_{m}^{-}}=(G)-\left(D_{2 m}^{d}\right)$ for $m \in \mathbb{N}$. It follows directly from the properties of the Burnside ring multiplication that if $\mathfrak{m}_{m}$ 
is odd, then $G-\operatorname{deg}(\mathscr{F}, \Omega) \neq 0$. More precisely, there exists an $m^{\prime} \in \mathbb{N}$ such that $m$ divides $m^{\prime}$ and $\operatorname{coeff}^{D_{2 m^{\prime}}^{d}}(G-\operatorname{deg}(\mathscr{A}, B(\mathscr{H})) \neq 0$ (for more details pertinent to this fact, we refer to Section 4. where a more involved symmetric setting is discussed). Therefore, by the existence property of the Brouwer equivariant degree, there exists a non-zero solution $u$ to the equation $\mathscr{F}(u)=0$ such that $G_{u} \geq D_{2 m^{\prime}}^{d}$. Clearly, such a solution $u$ is not a radial solution.

Let us point out that by applying an appropriate $H$-fixed point reduction, it is possible to remove the condition (D) from Theorem 3.1. To be more precise, put

$$
\mathscr{C}:=\left\{m \in \mathbb{Z}_{+}: \exists_{n \in \mathbb{N}} \exists_{\mu \in \sigma(A)} \text { such that } s_{n m}=\mu\right\} .
$$

Since $\mathscr{A}$ is a Fredholm operator, the set $\mathscr{C}$ is finite. Put $H_{l}:=\mathbb{Z}_{2 l}^{d}$. Then,

$$
\mathscr{H}^{H_{l}}=\overline{\bigoplus_{m=1}^{\infty} \mathscr{H}_{(2 m-1) l} .}
$$

Assume that there exists $l \in \mathbb{N}$ such that

$$
\mathscr{C} \cap\{(2 m-1) l: m \in \mathbb{N}\}=\emptyset .
$$

Then, $\mathscr{A}^{H_{l}}:=\left.\mathscr{A}\right|_{\mathscr{E}^{H_{l}}}: \mathscr{H}^{H_{l}} \rightarrow \mathscr{H}^{H_{l}}$ is an isomorphism and we obtain the following theorem.

Theorem 3.2. Under the assumptions $\left(A_{1}\right)\left(A_{4}\right)$, let $l \in \mathbb{N}$ be an integer such that (20) is satisfied. If there exists $m \in \mathbb{N}$ such that $\mathfrak{m}_{(2 m-1) l}$ is odd, then system (11) admits a non-radial solution.

Proof. One needs to repeat the same arguments that were presented in the proof of Theorem 3.1 .

\section{Semilinear Elliptic Systems on a Disc with Additional Symmetries}

(a) Symmetrically Interacting Systems. Let us consider a system composed of $n$-coupled identical systems of type (11) which we can write as follows:

$$
\left\{\begin{array}{l}
-\triangle u_{1}=f\left(z, u_{1}\right)+g_{1}\left(u_{1}, u\right), \\
-\triangle u_{2}=f\left(z, u_{2}\right)+g_{2}\left(u_{2}, u\right), \\
\vdots \\
-\triangle u_{n}=f\left(z, u_{n}\right)+g_{n}\left(u_{n}, u\right), \\
\left.u_{1}\right|_{\partial D}=\left.u_{2}\right|_{\partial D}=\cdots=\left.u_{n}\right|_{\partial D}=0,
\end{array}\right.
$$

where $f$ is similar to $\boldsymbol{f}$ in (1) $, u_{j}(z) \in \mathbb{R}^{s}, u=\left(u_{1}, u_{2}, \ldots, u_{n}\right)$ and the functions $g_{j}\left(u_{j}, u\right)$ describe the interaction between the $j$-th function $u_{j}$ and other functions. As an example, consider a configuration of 8 such systems coupled in a cube fashion (see figure below). Then system (21) can be written as:

$$
\left\{\begin{array}{l}
-\triangle u_{1}=f\left(z, u_{1}\right)+g\left(u_{2}-u_{1}, u_{4}-u_{1}, u_{6}-u_{1}\right) \\
-\triangle u_{2}=f\left(z, u_{2}\right)+g\left(u_{1}-u_{2}, u_{3}-u_{2}, u_{7}-u_{2}\right) \\
-\triangle u_{3}=f\left(z, u_{3}\right)+g\left(u_{2}-u_{3}, u_{4}-u_{3}, u_{8}-u_{3}\right) \\
-\triangle u_{4}=f\left(z, u_{4}\right)+g\left(u_{3}-u_{4}, u_{5}-u_{4}, u_{6}-u_{4}\right) \\
-\triangle u_{5}=f\left(z, u_{5}\right)+g\left(u_{4}-u_{5}, u_{6}-u_{5}, u_{8}-u_{5}\right) \\
-\triangle u_{6}=f\left(z, u_{6}\right)+g\left(u_{1}-u_{6}, u_{5}-u_{6}, u_{7}-u_{6}\right) \\
-\triangle u_{7}=f\left(z, u_{7}\right)+g\left(u_{2}-u_{7}, u_{6}-u_{7}, u_{8}-u_{7}\right), \\
-\triangle u_{8}=f\left(z, u_{8}\right)+g\left(u_{3}-u_{8}, u_{5}-u_{8}, u_{7}-u_{8}\right), \\
\left.u_{1}\right|_{\partial D}=\left.u_{2}\right|_{\partial D}=\cdots=\left.u_{8}\right|_{\partial D}=0
\end{array}\right.
$$


where the continuous function $g: \mathbb{R}^{s} \times \mathbb{R}^{s} \times \mathbb{R}^{s} \rightarrow \mathbb{R}^{s}$ is such that $g\left(x_{1}, x_{2}, x_{3}\right)=g\left(x_{2}, x_{3}, x_{1}\right)=$ $g\left(x_{1}, x_{3}, x_{2}\right)$ for all $x_{1}, x_{2}, x_{3} \in \mathbb{R}^{k}$ and $g(0,0,0)=0$. We will also assume that $g$ is differentiable at $(0,0,0)$, thus there exists an $s \times s$-matrix $B$ such that

$$
D g(0,0,0)\left(x_{1}, x_{2}, x_{3}\right)=B\left(x_{1}+x_{2}+x_{3}\right), \quad x_{1}, x_{2}, x_{3} \in \mathbb{R}^{s} .
$$

Keeping the exemplary system (22) in mind, assume that $\Gamma$ is a subgroup of the permutation group $S_{k}$ acting on $V:=\mathbb{R}^{k}$ by permuting the coordinates of vectors in $\mathbb{R}^{k}$. Consider system (1) assuming, in addition to conditions $\left(A_{1}\right)\left(A_{4}\right)$, that the following condition is satisfied:

$\left(B_{1}\right)$ the function $\boldsymbol{f}$ is $\Gamma$-equivariant, i.e. for every $\gamma \in \Gamma$ and $x \in V, \boldsymbol{f}(z, \gamma x)=\gamma \boldsymbol{f}(z, x)$ (for all $z \in D$ ).

We are interested in extending Theorems 3.1 and 3.2 to the symmetric setting providing an additional information on symmetric properties of non-radial solutions.

(b) Equivariant Setting in Functional Spaces. Put $G:=O(2) \times \Gamma \times \mathbb{Z}_{2}$ and consider the Hilbert $G$-representation $\mathscr{H}:=H_{o}^{2}(D ; V)$, with the $O(2) \times \mathbb{Z}_{2}$-action given by (18) and the $\Gamma$ action given by $(\gamma u)(z):=\gamma u(z), \gamma \in \Gamma, z \in D, u \in \mathscr{H}$. Then, the operator $\mathscr{F}$ given by (13) is $G$-equivariant.

Consider the $\Gamma$-isotypic decomposition of $V$

$$
V=V_{0} \oplus V_{1} \oplus V_{2} \oplus \cdots \oplus V_{\mathfrak{r}},
$$

where the $\Gamma$-isotypic component $V_{j}$ is modeled on the irreducible $\Gamma$-representation $\mathcal{U}_{j}$. We also put

$$
m_{j}:=\operatorname{dim} V_{j} / \operatorname{dim} \mathcal{U}_{j}, \quad j \in\{0,1, \ldots, \mathfrak{r}\} .
$$

To simplify the computations, we introduce an additional condition (cf. condition $\left(A_{3}\right)$ ):

$\left(B_{2}\right)$ For every $j \in\{0,1,2, \ldots, \mathfrak{r}\},\left.A\right|_{V_{j}}=\mu_{j} \operatorname{Id}_{V_{j}}$, for some $\mu_{j} \in \mathbb{R}$.

The $G$-isotypic decomposition of $\mathscr{H}$ can be easily constructed. For every $j \in\{0,1,2, \ldots, \mathfrak{r}\}$ and $(n, m) \in \mathbb{N} \times \mathbb{N} \cup\{0\}$ we put

$$
\mathscr{E}_{n m}^{j}:=\left\{J_{m}\left(s_{n m} r\right)(\cos (m \theta) \vec{a}+\sin (m \theta) \vec{b}): \vec{a}, \vec{b} \in V_{j}\right\},
$$

and

$$
\mathscr{H}_{m, j}:=\overline{\bigoplus_{n=1}^{\infty} \mathscr{E}_{n m}^{j}} .
$$

Then, we obtain the following $G$-isotypic decomposition of $\mathscr{H}$ :

$$
\mathscr{H}=\bigoplus_{j=0}^{\mathfrak{r}} \overline{\bigoplus_{m=0}^{\infty} \mathscr{H}_{m, j}}
$$

(here the $G$-isotypic component $\mathscr{H}_{m, j}$ is modeled on the irreducible $G$-representation $\mathcal{V}_{m, j}^{-}=\mathcal{W}_{m} \otimes$ $\mathcal{U}_{j}^{-}$, where $\mathcal{W}_{m}$ stands for the $m$-th irreducible $O(2)$-representation and $\mathcal{U}_{j}^{-}$is the representation $\mathcal{U}_{j}$ with the antipodal $\mathbb{Z}_{2}$-action).

In order to formulate and prove one of our main results in the symmetric setting, let us discuss some additional properties of the basic degrees. To begin with, take $\nu \in \mathbb{N}$ and define the Lie group homomorphism $\psi_{\nu}: O(2) \times \Gamma \times \mathbb{Z}_{2} \rightarrow O(2) \times \Gamma \times \mathbb{Z}_{2}$ by

$$
\psi_{\nu}\left(e^{i \theta}, \gamma, \pm 1\right)=\left(e^{i \nu \theta}, \gamma, \pm 1\right), \quad \psi_{\nu}\left(e^{i \theta} \kappa, \gamma, \pm 1\right)=\left(e^{i \nu \theta} \kappa, \gamma, \pm 1\right)
$$


(sometimes $\psi_{\nu}$ is called a $\nu$-folding homomorphism). This homomorphism induces the Burnside ring homomorphism $\Psi_{\nu}: A_{0}(G) \rightarrow A_{0}(G)$, where for a generator $(H) \in \Phi_{0}(G)$, one has

$$
\Psi_{\nu}(H)=\left(H_{\nu}\right), \quad H_{\nu}=\psi_{\nu}^{-1}(H) .
$$

In particular, for any $j=\{0,1, \ldots, \mathfrak{r}\}$ and $m \geq 0$, one has

$$
\Psi_{\nu}\left(\operatorname{deg}_{\mathcal{V}_{m, j}^{-}}\right)=\operatorname{deg}_{\mathcal{V}_{\nu m, j}^{-}}
$$

Remark 4.1. If $(H)$ is a maximal orbit type in $\mathscr{H} \backslash\{0\}$, then necessarily one has that $(H)$ is an orbit type in $\mathscr{H}_{0, j} \backslash\{0\}$ for some $j \in\{1, \ldots, \mathfrak{r}\}$. Hence, in this case, $(H)$ is an orbit type of a radially symmetric map (by the same token, $\Phi_{\nu}(H)=(H)$ ). Hence, there do not exist maximal orbit types in $\mathscr{H} \backslash\{0\}$ corresponding to a non-radial map. This suggests to consider maximal orbit types in $\mathscr{H}_{m, j} \backslash\{0\}$ with $m>0$.

For $m>0$, denote by $\mathfrak{M}_{m}$ the set of all maximal orbit types $(H)$ in $\Phi_{0}\left(G ; \mathscr{H}_{m} \backslash\{0\}\right)$. Clearly, $\Psi_{\nu}\left(\mathfrak{M}_{m}\right)=\mathfrak{M}_{m \nu}$ for any $\nu \in \mathbb{N}$ (in particular, $\Psi_{\nu}\left(\mathfrak{M}_{1}\right)=\mathfrak{M}_{\nu}$ ). Being motivated by Remark 4.1 and formula (19), and in order to simplify our exposition, consider the isotypic decomposition

$$
\mathscr{H}_{1}:=\mathscr{H}_{1,0} \oplus \mathscr{H}_{1,1} \oplus \cdots \oplus \mathscr{H}_{1, \mathfrak{r}} .
$$

Decomposition (28) together with formula (26) (see also (6), (223) and condition $\left(\mathrm{B}_{2}\right)$ ) allow us to refine formulas (16) and (17) as follows: for a given $(H) \in \mathfrak{M}_{1}$ and $\nu \in \mathbb{N}$, define

$$
\mathfrak{n}_{j}\left(H_{\nu}\right):=\left|\left\{n \in \mathbb{N}: s_{n \nu}<\mu_{j}, \operatorname{coeff}^{H_{\nu}}\left(\operatorname{deg}_{\mathcal{V}_{\nu, j}^{-}}\right) \neq 0\right\}\right|,
$$

and

$$
\mathfrak{m}\left(H_{\nu}\right):=\sum_{j=0}^{\mathfrak{r}} \mathfrak{n}_{j}\left(H_{\nu}\right) m_{j} .
$$

We are now in a position to formulate our first main result in the symmetric setting.

Theorem 4.2. Under the assumptions $\left(A_{1}\right)\left(A_{4}\right)$, $(D),\left(B_{1}\right)-\left(B_{2}\right)$, suppose that there exist $(H) \in$ $\mathfrak{M}_{1}$ and $\nu>0$ such that $\mathfrak{m}\left(H_{\nu}\right)$ is odd (cf. (29)-(30). Put

$$
\nu_{o}=\nu_{o}(H):=\max \left\{\nu^{\prime}: \mathfrak{m}\left(H_{\nu^{\prime}}\right) \text { is odd }\right\}
$$

(obviously, $\left.\nu_{o}<\infty\right)$. Then, system (1) admits a non-radial solution $u \in \mathscr{H}$ such that $G_{u}=H_{\nu_{o}}$ for some $r \in \mathbb{N}$.

Proof. Let $B_{\varepsilon}(0), B_{R}(0)$ and $\Omega$ be the same as in the proof of Theorem 3.1. Then, $\mathscr{F}$ is $B_{\varepsilon}(0)$-admissibly $G$-equivariantly homotopic to the isomorphism $\mathscr{A}$ and $B_{R}(0)$-admissibly $G$ equivariantly homotopic to Id. Moreover, formula (19) refines to the following one:

$$
\begin{aligned}
G-\operatorname{deg}(\mathscr{F}, \Omega) & =G-\operatorname{deg}\left(\mathscr{F}, B_{R}(0)\right)-G-\operatorname{deg}\left(\mathscr{F}, B_{\varepsilon}(0)\right) \\
& =(G)-\prod_{j=0}^{\mathfrak{r}} \prod_{m=0}^{\infty} \prod_{n=1}^{\infty} G-\operatorname{deg}\left(\mathscr{A}_{n, m}^{j}, B\left(\mathscr{E}_{n m}^{j}\right)\right),
\end{aligned}
$$

where $\mathscr{A}_{n, m}^{j}:=\left.\mathscr{A}\right|_{\mathscr{E}_{n m}^{j} j}$. Notice that

$$
G-\operatorname{deg}\left(\mathscr{A}_{n, m}^{j}, B\left(\mathscr{E}_{n m}^{j}\right)\right)= \begin{cases}\left(\operatorname{deg}_{\mathcal{V}_{m, j}^{-}}\right)^{m_{j}} & \text { if } s_{n m}<\mu_{j} \\ (G) & \text { otherwise }\end{cases}
$$


Also, for any basic degree $\operatorname{deg}_{\mathcal{V}_{m, j}^{-}}$and maximal orbit type $\left(H_{o}\right)$ in $\mathcal{V}_{m, j}^{-} \backslash\{0\}$, the recurrence formula (5) implies

$$
\operatorname{deg}_{\mathcal{V}_{m, j}^{-}}=(G)-x_{o}\left(H_{o}\right)+c, \quad-x_{o}:=\frac{(-1)^{\operatorname{dim} \mathcal{V}_{m, j}^{-H_{o}}}-1}{\left|W\left(H_{o}\right)\right|}
$$

where $c \in A(G)$ satisfies the condition: $\operatorname{coeff}^{H_{o}}(c)=0$. Then, by (34), one has

$$
x_{o}= \begin{cases}0 & \text { if } \operatorname{dim} \mathcal{V}_{m, j}^{-H_{o}} \text { is even; } \\ 1 & \text { if } \operatorname{dim} \mathcal{V}_{m, j}^{-H_{o}} \text { is odd and }\left|W\left(H_{o}\right)\right|=2 \\ 2 & \text { if } \operatorname{dim} \mathcal{V}_{m, j}^{-H_{o}} \text { is odd and }\left|W\left(H_{o}\right)\right|=1\end{cases}
$$

To complete the proof of Theorem 4.2, we need the following important

Lemma 4.3. Suppose that $\left(H_{o}\right)$ is a maximal orbit type in $\mathcal{V}_{m, j}^{-} \backslash\{0\}$ and $\mathcal{V}_{m^{\prime}, j^{\prime}}^{-} \backslash\{0\}$ and both $\mathcal{V}_{m, j}^{-H_{o}}$ and $\mathcal{V}_{m^{\prime}, j^{\prime}}^{-H_{o}}$ are of odd dimension. Then:

(i) $\operatorname{coeff}^{H_{o}}\left(\operatorname{deg} \mathcal{V}_{m, j}^{-}\right)=\operatorname{coeff}^{H_{o}}\left(\operatorname{deg} \mathcal{V}_{m^{\prime}, j^{\prime}}^{-}\right)$;

(ii) $\operatorname{coeff}^{H_{o}}\left(\operatorname{deg} \mathcal{V}_{m, j}^{-} \cdot \operatorname{deg} \mathcal{V}_{m^{\prime}, j^{\prime}}^{-}\right)=0$.

Proof of Lemma 4.3:

(i) Follows immediately from (35).

(ii) Consider the product

$$
\begin{aligned}
\operatorname{deg} \mathcal{V}_{m, j}^{-} \cdot \operatorname{deg} \mathcal{V}_{m^{\prime}, j^{\prime}}^{-} & =\left((G)-x_{o}\left(H_{o}\right)+c\right) \cdot\left((G)-x_{o}\left(H_{o}\right)+c^{\prime}\right) \\
& =(G)-2 x_{o}\left(H_{o}\right)+x_{o}^{2}\left(H_{o}\right) \cdot\left(H_{o}\right)+b,
\end{aligned}
$$

where $c, c^{\prime}, b \in A(G)$ satisfy $\operatorname{coeff}^{H_{o}}(c)=\operatorname{coeff}^{H_{o}}\left(c^{\prime}\right)=\operatorname{coeff}^{H_{o}}(b)=0$. Then, by using the recurrence formula (5), one obtains:

$$
\left(H_{o}\right) \cdot\left(H_{o}\right)=y_{o}\left(H_{o}\right)+d, \quad y_{o}:=\frac{n\left(H_{o}, H_{o}\right)^{2}\left|W\left(H_{o}\right)\right|^{2}}{\left|W\left(H_{o}\right)\right|}=\mid\left(W\left(H_{o}\right) \mid,\right.
$$

with $\operatorname{coeff}^{H_{o}}(d)=0$. Hence,

$$
-2 x_{o}\left(H_{o}\right)+x_{o}^{2}\left(H_{o}\right) \cdot\left(H_{o}\right)=\left\{\begin{array}{lll}
-2+2 & \text { if } x_{o}=1, & \left|W\left(H_{o}\right)\right|=2 \\
-4+4 & \text { if } x_{o}=2, & \left|W\left(H_{o}\right)\right|=1
\end{array} \cdot\left(H_{o}\right)=0,\right.
$$

and the statement follows.

Completion of the proof of Theorem 4.2. By (32)-(34) (see also (38)), one has:

$$
\prod_{j=0}^{\mathfrak{r}} \prod_{m=0}^{\infty} \prod_{n=1}^{\infty} G-\operatorname{deg}\left(\mathscr{A}_{n, m}^{j}, B\left(\mathscr{E}_{n m}^{j}\right)\right)=((G)-a) \cdot \prod_{k=1}^{\mathfrak{m}\left(H_{\nu_{o}}\right)}\left((G)+x_{o}\left(H_{\nu_{o}}\right)+c_{k}\right),
$$

where coeff ${ }^{H_{\nu_{o}}}\left(c_{k}\right)=\operatorname{coeff}^{H_{\nu_{o}}}(a)=0$. Since $\mathfrak{m}\left(H_{\nu_{o}}\right)$ is odd, Lemma 4.3 yields:

$$
\begin{aligned}
\prod_{k=1}^{\mathfrak{m}\left(H_{\nu_{o}}\right)}\left((G)+x_{o}\left(H_{\nu_{o}}\right)+c_{k}\right) & =\prod_{k=2}^{\mathfrak{m}\left(H_{\nu_{o}}\right)}\left((G)+x_{o}\left(H_{\nu_{o}}\right)+c_{k}\right) \cdot\left((G)+x_{o}\left(H_{\nu_{o}}\right)+c_{1}\right) \\
& =\left((G)-a^{\prime}\right) \cdot\left((G)+x_{o}\left(H_{\nu_{o}}\right)+c_{1}\right),
\end{aligned}
$$

where coeff ${ }^{H_{\nu_{o}}}\left(a^{\prime}\right)=0$. Combining (32), (36) and (37) with the maximality of $\left(H_{\nu_{o}}\right)$ yields:

$$
\begin{aligned}
G-\operatorname{deg}(\mathscr{F}, \Omega) & =(G)-((G)-b) \cdot\left((G)+x_{o}\left(H_{\nu_{o}}\right)+c_{1}\right) \\
& =x_{o}\left(H_{\nu_{o}}\right)-b-x_{o}\left(H_{\nu_{o}}\right) \cdot b-b-b \cdot c_{1}+c_{1}=x_{o}\left(H_{\nu_{o}}\right)+d,
\end{aligned}
$$


(here coeff ${ }^{H_{\nu_{o}}}(b)=$ coeff $\left.^{H_{\nu_{o}}}(d)=0\right)$. Therefore, by the existence property of the Brouwer equivariant degree, there exists a non-zero solution $u$ to the equation $\mathscr{F}(u)=0$ such that $G_{u} \geq H_{\nu_{o}}$. Clearly, such a solution $u$ is not a radial solution.

Similarly to the non-equivariant case, one can formulate the second main result providing the existence of non-radial solutions without assuming the condition (D).

Theorem 4.4. Under the assumptions $\left(A_{1}\right)\left(A_{4}\right),\left(B_{1}\right)-\left(B_{2}\right)$, let $l \in \mathbb{N}$ be an integer such that (20) is satisfied. Suppose, in addition, that there exist $(H) \in \mathfrak{M}_{1}$ and $m>0$ such that $\mathfrak{m}\left(H_{(2 m-1) l}\right)$ is odd (cf. (29)-(30). Put

$$
\nu_{o}:=\max \left\{(2 m-1) l: \mathfrak{m}\left(H_{(2 m-1) l}\right) \text { is odd }\right\}
$$

(obviously, $\nu_{o}<\infty$ ). Then, system (11) admits a non-radial solution $u \in \mathscr{H}$ such that $G_{u}=H_{\nu_{o}}$ for some $r \in \mathbb{N}$.

(c) Example. Assume that $\Gamma=S_{4}$ acts on the space $V:=\mathbb{R}^{8}$ by permuting the coordinates of vectors the same way as the symmetries of a cube permute its vertices. We consider system (11) for which the map $\boldsymbol{f}$ satisfies conditions $\left(A_{1}\right)\left(A_{4}\right)$, $\left(\mathrm{B}_{1}\right)$, and the matrix $A$ is given by

$$
A=\left[\begin{array}{llllllll}
c & d & 0 & d & 0 & d & 0 & 0 \\
d & c & d & 0 & 0 & 0 & d & 0 \\
0 & d & c & d & 0 & 0 & 0 & d \\
d & 0 & d & c & d & 0 & 0 & 0 \\
0 & 0 & 0 & d & c & d & 0 & d \\
d & 0 & 0 & 0 & d & c & d & 0 \\
0 & d & 0 & 0 & 0 & d & c & d \\
0 & 0 & d & 0 & d & 0 & d & c
\end{array}\right]
$$

(the values of $c$ and $d$ will be specified later on). Notice that one has the following table of characters:

\begin{tabular}{|c|ccccc|}
\hline$\chi$ & () & $(1,2)$ & $(1,2)(3,4)$ & $(1,2,3)$ & $(1,2,3,4)$ \\
\hline$\chi_{0}$ & 1 & 1 & 1 & 1 & 1 \\
$\chi_{1}$ & 1 & -1 & 1 & 1 & -1 \\
$\chi_{2}$ & 2 & 0 & 2 & -1 & 0 \\
$\chi_{3}$ & 3 & 1 & -1 & 0 & -1 \\
$\chi_{4}$ & 3 & -1 & -1 & 0 & 1 \\
\hline$\chi_{V}$ & 8 & 0 & 0 & 2 & 0 \\
\hline
\end{tabular}

which implies that

$$
V=\mathcal{U}_{0} \oplus \mathcal{U}_{1} \oplus \mathcal{U}_{3} \oplus \mathcal{U}_{4}
$$

(here $\mathcal{U}_{j}$ stands for the irreducible representation corresponding to the character $\chi_{j}$ ). Clearly, (40) implies condition $\left(\mathrm{B}_{2}\right)$. One can easily compute the spectrum of $A$ :

$$
\sigma(A)=\left\{\mu_{0}=c+3 d, \mu_{1}=c-3 d, \mu_{3}=c+d, \mu_{4}=c-d\right\} .
$$

Obviously,

$$
\operatorname{dim} E\left(\mu_{0}\right)=\operatorname{dim} E\left(\mu_{1}\right)=1, \quad \text { and } \operatorname{dim} E\left(\mu_{3}\right)=\operatorname{dim} E\left(\mu_{4}\right)=3
$$

and

$$
E\left(\mu_{0}\right)=\mathcal{U}_{0}, \quad E\left(\mu_{1}\right)=\mathcal{U}_{1}, \quad E\left(\mu_{3}\right)=\mathcal{U}_{3}, \quad E\left(\mu_{4}\right)=\mathcal{U}_{4}
$$

The numbers $s_{n m}$ are shown in the table below: 


\begin{tabular}{|c|cccccc|}
\hline$n$ & $J_{0}(x)$ & $J_{1}(x)$ & $J_{2}(x)$ & $J_{3}(x)$ & $J_{4}(x)$ & $J_{5}(x)$ \\
\hline 1 & 2.4048 & 3.8317 & 5.1356 & 6.3802 & 7.5883 & 8.7715 \\
2 & 5.5201 & 7.0156 & 8.4172 & 9.7610 & 11.0647 & 12.3386 \\
3 & 8.6537 & 10.1735 & 11.6198 & 13.0152 & 14.3725 & 15.7002 \\
4 & 11.7915 & 13.3237 & 14.7960 & 16.2235 & 17.6160 & 18.9801 \\
5 & 14.9309 & 16.4706 & 17.9598 & 19.4094 & 20.8269 & 22.2178 \\
\hline
\end{tabular}

Take $c=4$ and $d=1$, thus $\mu_{0}=7, \mu_{1}=1, \mu_{3}=5, \mu_{4}=3$ and condition (D) is satisfied.

Put $G:=O(2) \times S_{4} \times \mathbb{Z}_{2}$. Then,

$$
\begin{aligned}
G-\operatorname{deg}(\mathscr{F}, \Omega) & =(G)-\operatorname{deg}_{\mathcal{V}_{0,0}^{-}}^{2} \cdot \operatorname{deg}_{\mathcal{V}_{1,0}^{-}} \cdot \operatorname{deg}_{\mathcal{V}_{2,0}^{-}} \cdot \operatorname{deg}_{\mathcal{V}_{3,0}^{-}} \cdot \operatorname{deg}_{\mathcal{V}_{0,3}^{-}} \cdot \operatorname{deg}_{\mathcal{V}_{1,3}^{-}} \cdot \operatorname{deg}_{\mathcal{V}_{0,4}^{-}} \\
& =(G)-\operatorname{deg}_{\mathcal{V}_{1,0}^{-}} \cdot \operatorname{deg}_{\mathcal{V}_{2,0}^{-}} \cdot \operatorname{deg}_{\mathcal{V}_{3,0}^{-}} \cdot \operatorname{deg}_{\mathcal{V}_{0,3}^{-}} \cdot \operatorname{deg}_{\mathcal{V}_{1,3}^{-}} \cdot \operatorname{deg}_{\mathcal{V}_{0,4}^{-}} \cdot
\end{aligned}
$$

In order to effectively apply Theorem4.2, we used GAP package EquiDeg to carry on all the related symbolic computations. We refer to Appendix B for the exact GAP codes that were applied for this example. In particular, the maximal orbit types in $\mathfrak{M}_{1}$ are:

$$
\begin{array}{ll}
\left(H_{1,228}\right)=\left(D_{6} \times{ }_{D_{6}} D_{3}^{p}\right), & \left(H_{1,248}\right)=\left(D_{4} \times{ }_{D_{4}}^{\mathbb{Z}_{2}^{-}} D_{4}^{p}\right), \quad\left(H_{1,286}\right)=\left(D_{2}^{D_{1}} \times \times_{\mathbb{Z}_{2}}^{D_{2}^{d}} D_{2}^{p}\right), \\
\left(H_{1,334}\right)=\left(D_{2}^{D_{1}} \times_{\mathbb{Z}_{2}}^{D_{4}^{z}} D_{4}^{p}\right), & \left(H_{1,333}\right)=\left(D_{2}^{D_{1}} \times_{\mathbb{Z}_{2}}^{D_{4}^{d}} D_{4}^{p}\right), \quad\left(H_{1,360}\right)=\left(D_{2}^{D_{1}} \times_{\mathbb{Z}_{2}}^{S_{4}} S_{4}^{p}\right), \\
\left(H_{1,359}\right)=\left(D_{2}^{D_{1}} \times_{\mathbb{Z}_{2}}^{S_{4}^{-}} S_{4}^{p}\right) . &
\end{array}
$$

Remark 4.5. (i) For any subgroup $S \leq S_{4}$, the symbol $S^{p}$ stands for $S \times \mathbb{Z}_{2}$.

(ii) Given two subgroups $H \leq O(2)$ and $K \leq S_{4}^{p}$, we refer to Appendix $\mathrm{A}$, item (a), for the "amalgamated notation" $H^{R} \times{ }_{L}^{K} Z$.

(iii) We refer to [2] for the explicit description of the (sub)groups $S_{4}^{-}, D_{k}^{z}, D_{k}^{d}$, and $\mathbb{Z}_{2}^{-}$.

Moreover, we have

$$
\begin{aligned}
\operatorname{deg}_{\mathcal{V}_{1,0}^{-}} & =(G)-\left(\boldsymbol{H}_{1,360}\right) \\
\operatorname{deg}_{\mathcal{V}_{1,3}^{-}} & =(G)-2\left(H_{1,46}\right)-\left(H_{1,83}\right)+2\left(H_{1,108}\right)+2\left(H_{1,126}\right)+2\left(H_{1,129}\right)+2\left(H_{1,204}\right) \\
& +\left(H_{1,212}\right)-2\left(\boldsymbol{H}_{\mathbf{1 , 2 2 8}}\right)-2\left(\boldsymbol{H}_{\mathbf{1 , 2 4 8}}\right)-\left(\boldsymbol{H}_{\mathbf{1 , 2 8 6}}\right)-\left(H_{1,330}\right)-\left(\boldsymbol{H}_{\mathbf{1 , 3 3 4}}\right),
\end{aligned}
$$

where we denote in bold the maximal orbit types from $\mathfrak{M}_{1}$. Then, by inspection, one can easily determine that

$$
\mathfrak{m}\left(\boldsymbol{H}_{1,360}\right)=1, \quad \mathfrak{m}\left(\boldsymbol{H}_{\mathbf{1 , 2 2 8}}\right)=1, \quad \mathfrak{m}\left(\boldsymbol{H}_{\mathbf{1 , 2 4 8}}\right)=1, \quad \mathfrak{m}\left(\boldsymbol{H}_{\mathbf{1 , 2 8 6}}\right)=1, \quad \mathfrak{m}\left(\boldsymbol{H}_{\mathbf{1 , 3 3 4}}\right)=1
$$


Since, the exact degree $G$ - $\operatorname{deg}(\mathscr{F}, \Omega)$ can be effectively computed using the GAP package, we can use it to double check the correctness of our conclusions:

$$
\begin{aligned}
G-\operatorname{deg}(\mathscr{F}, \Omega) & =-2\left(H_{1,2}\right)-2\left(H_{1,5}\right)-2\left(H_{1,8}\right)-8\left(H_{1,11}\right)-2\left(H_{1,14}\right)+4\left(H_{1,20}\right) \\
& \left.+4\left(H_{1,21}\right)+2\left(H_{1,25}\right)+2\left(H_{1,41}\right)+2\left(H_{1,46}\right)\right]+4\left(H_{1,51}\right)+2\left(H_{1,53}\right) \\
& +2\left(H_{1,57}\right)+2\left(H_{1,59}\right)-2\left(H_{1,77}\right)+1\left(H_{1,83}\right)-\left(H_{1,88}\right)-\left(H_{1,89}\right)+2\left(H_{1,90}\right) \\
& \left.+\left(H_{1,91}\right)\right)-\left(H_{1,92}\right)+2\left(H_{1,98}\right)+2\left(H_{1,102}\right)+2\left(H_{1,103}\right)-\left(H_{1,106}\right) \\
& -2\left(H_{1,108}\right)-2\left(H_{1,115}\right)-2\left(H_{1,117}\right)-2\left(H_{1,119}\right)-2\left(H_{1,126}\right)-2\left(H_{1,129}\right) \\
& +2\left(H_{1,152}\right)+2\left(H_{1,155}\right)-2\left(H_{1,204}\right)-\left(H_{1,208}\right)-\left(H_{1,212}\right)+\left(H_{1,220}\right) \\
& +\left(H_{1,221}\right)-\left(H_{1,223}\right)+\left(H_{1,224}\right)-2\left(H_{1,227}\right)-2\left(H_{1,228}\right)+\left(H_{1,244}\right)+\left(H_{1,245}\right) \\
& -\left(H_{1,246}\right)+2\left(H_{1,248}\right)-2\left(H_{1,256}\right)+\left(H_{1,286}\right)-\left(H_{1,292}\right)+\left(H_{1,295}\right) \\
& +\left(H_{1,298}\right)-\left(H_{1,300}\right)-\left(H_{1,301}\right)+\left(H_{1,330}\right)+\left(H_{1,334}\right)-\left(H_{1,360}\right)-\left(H_{2,17}\right) \\
& +\left(H_{2,88}\right)+\left(H_{2,89}\right)+\left(H_{2,106}\right)+\left(H_{2,223}\right)-\left(H_{2,244}\right)-\left(H_{2,245}\right)-\left(H_{2,295}\right) \\
& -\left(H_{2,298}\right)+\left(H_{2,360}\right)-\left(H_{3,17}\right)+\left(H_{3,88}\right)+\left(H_{3,89}\right)+\left(H_{3,106}\right)+\left(H_{3,223}\right) \\
& -\left(H_{3,244}\right)-\left(H_{3,245}\right)-\left(H_{3,295}\right)-\left(H_{3,298}\right)+\left(H_{3,360}\right)+\left(H_{0,12}\right)-\left(H_{0,43}\right) \\
& -\left(H_{0,44}\right)-\left(H_{0,52}\right)-\left(H_{0,90}\right)+\left(H_{0,98}\right)+\left(H_{0,99}\right)+\left(H_{0,110}\right)+\left(H_{0,111}\right) .
\end{aligned}
$$

On the other hand, by analyzing the coefficients of $G-\operatorname{deg}(\mathscr{F}, \Omega)$, one can also deduct the existence of various symmetric types of radial solutions. More precisely, the maximal orbit types in $\bigoplus_{j=0}^{\mathfrak{r}} \mathscr{H}_{0, j} \backslash\{0\}$ with non-zero coefficients in $G-\operatorname{deg}(\mathscr{F}, \Omega)$ are:

$$
\begin{aligned}
\left(H_{0,84}\right) & =\left(O(2) \times D_{2}^{d}\right), \quad\left(H_{0,98}\right)=\left(O(2) \times D_{3}\right), \quad\left(H_{0,99}\right)=\left(O(2) \times D_{3}^{z}\right) \\
\left(H_{0,110}\right) & =\left(O(2) \times D_{4}^{z}\right), \quad\left(H_{0,111}\right)=\left(O(2) \times D_{4}^{d}\right) .
\end{aligned}
$$

Combing this with (41) and Theorem 4.2, one obtains the following

Theorem 4.6. Under the assumptions $\left(A_{1}\right)\left(A_{4}\right),\left(B_{1}\right)-\left(B_{2}\right)$ assume that the matrix $A$ is given by (39) with $c=4$ and $d=1$. The system (11) admits at least five different orbits of non-radial solutions $u \in \mathscr{H}$ with the following orbit types $\left(G_{u}\right)$ :

- $\left(D_{6 m}^{\mathbb{Z}_{m}} \times_{D_{6}} D_{3}^{p}\right)$ for some $m \in \mathbb{N}$;

- $\left(D_{4 m}^{\mathbb{Z}_{m}} \times \frac{\mathbb{Z}_{2}^{-}}{D_{4}} D_{4}^{p}\right)$ for some $m \in \mathbb{N}$;

- $\left(D_{2 m}^{D_{m}} \times{ }_{\mathbb{Z}_{2}^{2}}^{D_{2}^{d}} D_{2}^{p}\right)$ for some $m \in \mathbb{N}$;

- $\left(D_{2 m}^{D_{m}} \times_{\mathbb{Z}_{2}}^{D_{4}^{z}} D_{4}^{p}\right)$ for some $m \in \mathbb{N}$;

- $\left(D_{2 m}^{D_{m}} \times_{\mathbb{Z}_{2}}^{S_{4}} S_{4}^{p}\right)$ for some $m \in \mathbb{N}$.

Moreover, the system (11) admits at least four different orbits of radial solutions $u \in \mathscr{H}$ with the following orbit types $\left(G_{u}\right)$ :

$$
\left(O(2) \times D_{3}\right), \quad\left(O(2) \times D_{3}^{z}\right), \quad\left(O(2) \times D_{4}^{z}\right), \quad\left(O(2) \times D_{4}^{d}\right) .
$$

Proof. The statement follows directly from Theorem 4.2. To be more precise, notice that for the orbit types $(H)$ with $H=H_{1,228}, H_{1,248}, H_{1,286}$ and $H_{1,334}$, we have $\nu=1$ and $\nu_{o}=1$, and for $H=H_{1,360}$, we have $\nu=1$ and $\nu_{o}=3$. On the other hand, for the maximal orbit types $\left(O(2) \times D_{3}\right),\left(O(2) \times D_{3}^{z}\right),\left(O(2) \times D_{4}^{z}\right)$ and $\left(O(2) \times D_{4}^{d}\right)$, one can conclude the existence of the related radial solutions to (1D) by a direct inspection of the explicit formula for $G$-deg $(\mathscr{F}, \Omega)$, where it is evident that all these orbit types appear with non-zero coefficients. 


\section{Appendix A. Equivariant Brouwer Degree Background}

(a) Amalgamated Notation. Given two groups $G_{1}$ and $G_{2}$, the well-known result of É. Goursat (see [11]) provides the following description of a subgroup $\mathscr{U} \leq G_{1} \times G_{2}$ : there exist subgroups $H \leq G_{1}$ and $K \leq G_{2}$, a group $L$, and two epimorphisms $\varphi: H \rightarrow L$ and $\psi: K \rightarrow L$ such that

$$
\mathscr{U}=\{(h, k) \in H \times K: \varphi(h)=\psi(k)\} .
$$

The widely used notation for $\mathscr{U}$ is

$$
\mathscr{U}:=H^{\varphi} \times{ }_{L}^{\psi} K,
$$

in which case $H^{\varphi} \times{ }_{L}^{\psi} K$ is called an amalgamated subgroup of $G_{1} \times G_{2}$.

In this paper, we are interested in describing conjugacy classes of $\mathscr{U}$. Therefore, to make notation (42) simpler and self-contained, it is enough to indicate $L, Z=\operatorname{Ker}(\varphi)$ and $R=\operatorname{Ker}(\psi)$. Hence, instead of (42), we use the following notation:

$$
\mathscr{U}=: H^{Z} \times{ }_{L}^{R} K \text {. }
$$

(b) Equivariant Notation. Below $\mathcal{G}$ stands for a compact Lie group. For a subgroup $H$ of $\mathcal{G}$, denote by $N(H)$ the normalizer of $H$ in $\mathcal{G}$ and by $W(H)=N(H) / H$ the Weyl group of $H$. The symbol $(H)$ stands for the conjugacy class of $H$ in $\mathcal{G}$. Put $\Phi(\mathcal{G}):=\{(H): H \leq \mathcal{G}\}$. The set $\Phi(\mathcal{G})$ has a natural partial order defined by $(H) \leq(K)$ iff $\exists g \in \mathcal{G} g H g^{-1} \leq K$. Put $\Phi_{0}(\mathcal{G}):=\{(H) \in \Phi(\mathcal{G}): W(H)$ is finite $\}$.

For a $\mathcal{G}$-space $X$ and $x \in X$, denote by $\mathcal{G}_{x}:=\{g \in \mathcal{G}: g x=x\}$ the isotropy group of $x$ and call $\left(\mathcal{G}_{x}\right)$ the orbit type of $x \in X$. Put $\Phi(\mathcal{G}, X):=\left\{(H) \in \Phi_{0}(\mathcal{G}):(H)=\left(\mathcal{G}_{x}\right)\right.$ for some $\left.x \in X\right\}$ and $\Phi_{0}(\mathcal{G}, X):=\Phi(\mathcal{G}, X) \cap \Phi_{0}(\mathcal{G})$. For a subgroup $H \leq \mathcal{G}$, the subspace $X^{H}:=\left\{x \in X: \mathcal{G}_{x} \geq H\right\}$ is called the $H$-fixed-point subspace of $X$. If $Y$ is another $\mathcal{G}$-space, then a continuous map $f: X \rightarrow Y$ is called equivariant if $f(g x)=g f(x)$ for each $x \in X$ and $g \in \mathcal{G}$. Let $V$ be a finite-dimensional $\mathcal{G}$-representation (without loss of generality, orthogonal). Then, $V$ decomposes into a direct sum

$$
V=V_{0} \oplus V_{1} \oplus \cdots \oplus V_{r},
$$

where each component $V_{i}$ is modeled on the irreducible $\mathcal{G}$-representation $\mathcal{V}_{i}, i=0,1,2, \ldots, r$, that is, $V_{i}$ contains all the irreducible subrepresentations of $V$ equivalent to $\mathcal{V}_{i}$. Decomposition (44) is called $\mathcal{G}$-isotypic decomposition of $V$.

(b) Axioms of Equivariant Brouwer Degree. Denote by $\mathcal{M}^{\mathcal{G}}$ the set of all admissible $\mathcal{G}$-pairs and let $A_{0}(\mathcal{G})$ stand for the Burnside ring of $\mathcal{G}$ (see Introduction, items (a) and (b) respectively). The following result (cf. 2]) can be considered as an axiomatic definition of the $\mathcal{G}$-equivariant Brouwer degree.

Theorem A.1. There exists a unique map $\mathcal{G}$ - $\operatorname{deg}: \mathcal{M}^{\mathcal{G}} \rightarrow A_{0}(\mathcal{G})$, which assigns to every admissible $\mathcal{G}$-pair $(f, \Omega)$ an element $G-\operatorname{deg}(f, \Omega) \in A_{0}(\mathcal{G})$

$$
\mathcal{G}-\operatorname{deg}(f, \Omega)=\sum_{(H)} n_{H}(H)=n_{H_{1}}\left(H_{1}\right)+\cdots+n_{H_{m}}\left(H_{m}\right),
$$

satisfying the following properties:

(Existence) If $\mathcal{G}-\operatorname{deg}(f, \Omega) \neq 0$, i.e., $n_{H_{i}} \neq 0$ for some $i$ in (45), then there exists $x \in \Omega$ such that $f(x)=0$ and $\left(\mathcal{G}_{x}\right) \geq\left(H_{i}\right)$.

(Additivity) Let $\Omega_{1}$ and $\Omega_{2}$ be two disjoint open $\mathcal{G}$-invariant subsets of $\Omega$ such that $f^{-1}(0) \cap \Omega \subset \Omega_{1} \cup \Omega_{2}$. Then,

$$
\mathcal{G}-\operatorname{deg}(f, \Omega)=\mathcal{G}-\operatorname{deg}\left(f, \Omega_{1}\right)+\mathcal{G}-\operatorname{deg}\left(f, \Omega_{2}\right) .
$$


(Homotopy) If $h:[0,1] \times V \rightarrow V$ is an $\Omega$-admissible $\mathcal{G}$-homotopy, then

$$
\mathcal{G}-\operatorname{deg}\left(h_{t}, \Omega\right)=\text { constant. }
$$

(Normalization) Let $\Omega$ be a G-invariant open bounded neighborhood of 0 in $V$. Then,

$$
\mathcal{G}-\operatorname{deg}(\operatorname{Id}, \Omega)=(\mathcal{G}) \text {. }
$$

(Multiplicativity) For any $\left(f_{1}, \Omega_{1}\right),\left(f_{2}, \Omega_{2}\right) \in \mathcal{M}^{\mathcal{G}}$,

$$
\mathcal{G}-\operatorname{deg}\left(f_{1} \times f_{2}, \Omega_{1} \times \Omega_{2}\right)=\mathcal{G}-\operatorname{deg}\left(f_{1}, \Omega_{1}\right) \cdot \mathcal{G}-\operatorname{deg}\left(f_{2}, \Omega_{2}\right),
$$

where the multiplication '. is taken in the Burnside ring $A_{0}(\mathcal{G})$.

(Recurrence Formula) For an admissible $\mathcal{G}$-pair $(f, \Omega)$, the $\mathcal{G}$-degree (45) can be computed using the following Recurrence Formula:

$$
n_{H}=\frac{\operatorname{deg}\left(f^{H}, \Omega^{H}\right)-\sum_{(K)>(H)} n_{K} n(H, K)|W(K)|}{|W(H)|},
$$

where $|X|$ stands for the number of elements in the set $X$ and $\operatorname{deg}\left(f^{H}, \Omega^{H}\right)$ is the Brouwer degree of the map $f^{H}:=\left.f\right|_{V^{H}}$ on the set $\Omega^{H} \subset V^{H}$.

The $G$ - $\operatorname{deg}(f, \Omega)$ is called the $\mathcal{G}$-equivariant Brouwer degree of $f$ in $\Omega$.

(c) Computation of Brouwer Equivariant Degree. Consider a $\mathcal{G}$-equivariant linear isomorphism $T: V \rightarrow V$ and assume that $V$ has a $\mathcal{G}$-isotypic decomposition (44). Then, by the Multiplicativity property,

$$
\mathcal{G}-\operatorname{deg}(T, B(V))=\prod_{i=0}^{r} \mathcal{G}-\operatorname{deg}\left(T_{i}, B\left(V_{i}\right)\right)=\prod_{i=0}^{r} \prod_{\mu \in \sigma_{-}(T)}\left(\operatorname{deg}_{\mathcal{V}_{i}}\right)^{m_{i}(\mu)}
$$

where $T_{i}=\left.T\right|_{V_{i}}, \sigma_{-}(T)$ denotes the real negative spectrum of $T$ (i.e., $\sigma_{-}(T)=\{\mu \in \sigma(T): \mu<0\}$ ) and $m_{i}(\mu)=\operatorname{dim}\left(E(\mu) \cap V_{i}\right)$ (here $E(\mu)$ stands for the generalized eigenspace of $T$ corresponding to $\mu$ ). Notice that the basic degrees can be effectively computed from (46):

$$
\operatorname{deg}_{\mathcal{V}_{i}}=\sum_{(H)} n_{H}(H)
$$

where

$$
n_{H}=\frac{(-1)^{\operatorname{dim} \mathcal{V}_{i}^{H}}-\sum_{H<K} n_{K} n(H, K)|W(K)|}{|W(H)|} .
$$

\section{Appendix B. GAP PACKAGE EquiDeg}

In this paper we used the GAP package EquiDeg, developed by Hao-Pin Wu. In order to generate the amalgamated notation for the subgroups of $G$ we applied in the GAP code the following abbreviations for the names of subgroups of $S_{4} \times \mathbb{Z}_{2}: \quad \mathrm{Z} 1:=\mathbb{Z}_{1}, \mathrm{Z} 2:=\mathbb{Z}_{2}, \mathrm{D} 1 \mathrm{z}:=D_{1}^{z}$, $\mathrm{D} 1:=D_{1}, \quad \mathrm{Z} 2 \mathrm{~m}:=\mathbb{Z}_{2}^{-}, \quad \mathrm{Z} 1 \mathrm{p}:=\mathbb{Z}_{1} \times \mathbb{Z}_{2}, \quad \mathrm{Z3}:=\mathbb{Z}_{3}, \quad \mathrm{Z} 2 \mathrm{p}:=\mathbb{Z}_{2} \times \mathbb{Z}_{2}, \quad \mathrm{~V} 4 \mathrm{~m}:=V_{4}^{-}, \quad \mathrm{D} 2:=D_{2}, \quad \mathrm{Z} 4:=$ $\mathbb{Z}_{4}, \mathrm{~V} 4:=V_{4}, \mathrm{D} 2 \mathrm{z}:=D_{2}^{z}, \mathrm{Z} 4 \mathrm{~d}:=\mathbb{Z}_{4}^{d}, \mathrm{D} 2 \mathrm{~d}:=D_{2}^{d}, \mathrm{D} 1 \mathrm{p}:=D_{1} \times \mathbb{Z}_{2}, \mathrm{Z3p}:=\mathbb{Z}_{3} \times \mathbb{Z}_{2}, \mathrm{D} 3:=D_{3}$, $\mathrm{D} 3 \mathrm{z}:=D_{3}^{z}, \mathrm{~V} 4 \mathrm{p}:=V_{4} \times \mathbb{Z}_{2}, \mathrm{D} 4 \mathrm{~d}:=D_{4}^{d}, \quad \mathrm{Z} 4 \mathrm{p}:=\mathbb{Z}_{4} \times \mathbb{Z}_{2}, \quad \mathrm{D} 4:=D_{4}, \mathrm{D} 2 \mathrm{p}:=D_{2} \times \mathbb{Z}_{2}, \quad \mathrm{D} 4 \mathrm{z}:=D_{4}^{z}$, D4hd: $=D_{4}^{\widehat{d}}, \quad \mathrm{D} 3 \mathrm{p}:=D_{3} \times \mathbb{Z}_{2}, \mathrm{~A} 4:=A_{4}, \mathrm{D} 4 \mathrm{p}:=D_{4} \times \mathbb{Z}_{2}, \mathrm{~S} 4:=S_{4}, \mathrm{~A} 4 \mathrm{p}:=A_{4} \times \mathbb{Z}_{2}, \mathrm{~S} 4 \mathrm{~m}:=S_{4}^{-}$, $\mathrm{S} 4 \mathrm{p}:=S_{4} \times \mathbb{Z}_{2}$, where we use the same notation as in [2], page 157 .

GAP code used in this paper: 


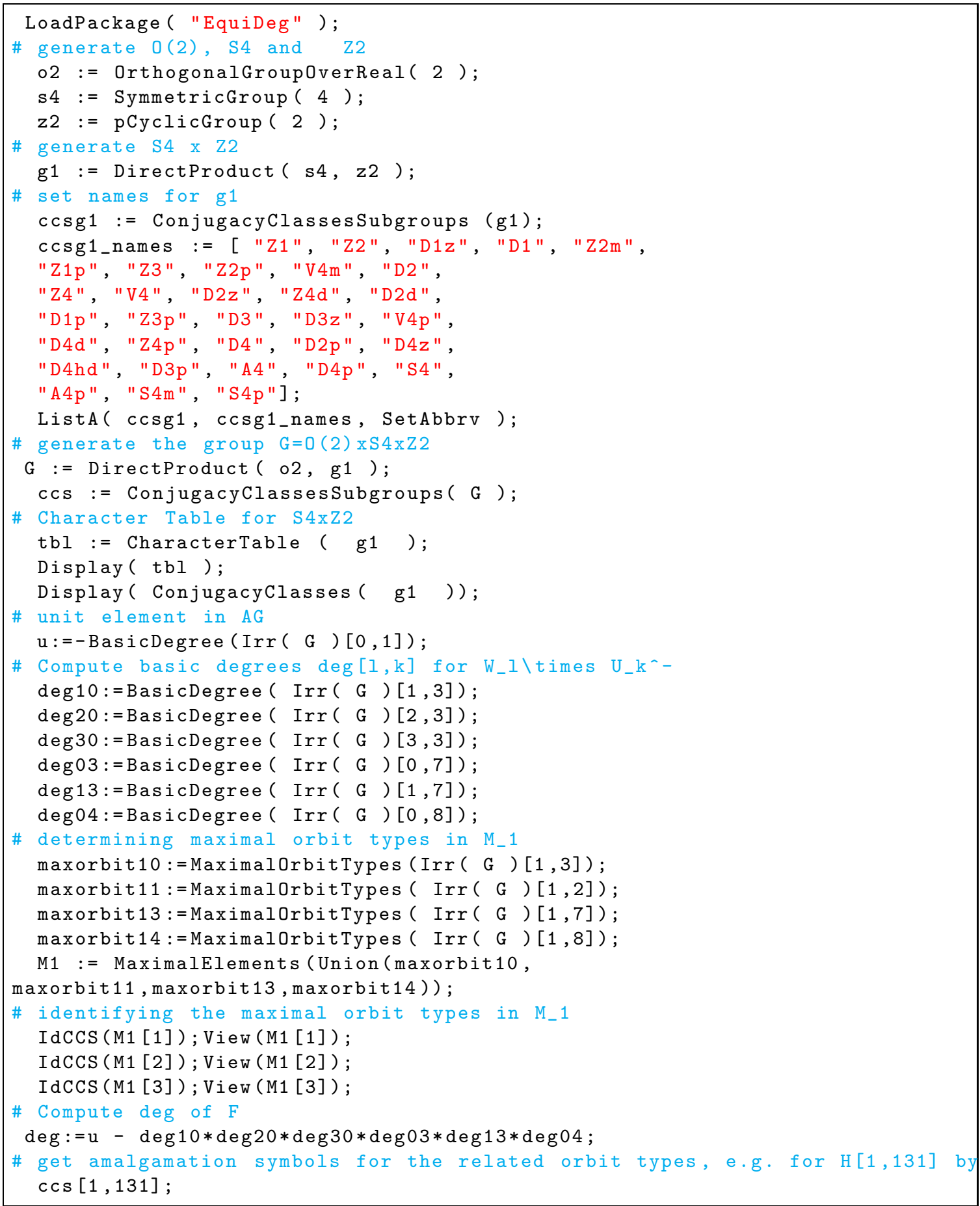

\section{REFERENCES}

[1] Z. Balanov, W. Krawcewicz, S. Rybicki and H. Steinlein. A short treatise on the equivariant degree theory and its applications, J.Fixed Point Theory App. 8:1-74, 2010. 
[2] Z. Balanov, W. Krawcewicz and H. Steinlein. Applied Equivariant Degree. AIMS Series on Differential Equations \& Dynamical Systems, Vol.1, 2006.

[3] T. Bartsch, N. Dancer and Z-Q. Wang, A Liouville theorem, a-priori bounds, and bifurcating branches of positive solutions for a nonlinear elliptic system, Calc. Var. (2010) 37: 345-361

[4] T. Bartsch and D. G. de Figueiredo, Infinitely many solutions of nonlinear elliptic systems, Topics in Nonlinear Analysis in Progress in Nonlinear Differential Equations and Their Applications book series, PNLDE, volume 35 (1999), pp 51-67.

[5] J. Bracho, M. Clapp and W. Marzantowicz, Symmetry breaking solutions of nonlinear elliptic system, Top. Meth. in Nonlinear Analysis, 26 (2005), 189-201.

[6] H. Brezis, Functional Analysis, Sobolev Spaces and Partial Differential Equations, Springer-Verlag, New York, 2010.

[7] M. Calanchi and B. Ruf, Radial and non radial solutions for Hardy-Hénon type elliptic systems, Calc. Var. (2010) 38: 111-133

[8] Ph. Clément, D.G. de Figueiredo and E.A. Mitidieri, A priori estimates for positive solutions of semilinear elliptic systems via Hardy-Sobolev inequalities. Pitman Res. Notes in Math. 1996, pp. 73-91.

[9] C. Cosner, Positive solutions For superlinear elliptic systems without variational structure, Nonlinear Anal. TMA. 1984, 8, 1427-1436.

[10] B. Gidas, W.M. Ni and L. Nirenberg, Symmetry and related properties via the maximum principle. Commun. Math. Phys. 68 (3), 209-243 (1979)

[11] É. Goursat, Sur les substitutions orthogonales et les divisions régulières de l'espace, Annales Scientifiques de l'École Normale Supérieure, 6 (1889), 9-102.

[12] M. K. Krasnoselskii, Topological methods in the theory of nonlinear integral equations (Pergamon Press, New York, 1964.

[13] M.A. Krasnosel'skii, P.P. Zabreiko, E.I. Pustylnik and P.E. Sobolevskii, Integral Operators in Spaces of Summable Functions. Noordhoff International Publishing, Leyden (1976).

[14] Z. Lou, T. Weth and Z. Zhang, Symmetry breaking via Morse index for equations and systems of HénonSchrödinger type, Z. Angew. Math. Phys. (2019) 70: 35

[15] M.S. Montenegro, Criticalidade, superlinearidade e sublinearidade para sistemas elíptico semilineares, Tese de Doutoramento, Unicamp, 1997.

[16] M.A.S. Souto, Sobre a existôncia de soluções positivas para sistemas cooperativos não-lineares, Tese de doutorado 1992, UNICAMP.

[17] T. Weth, Symmetry of solutions to variational problems for nonlinear elliptic equations via reflection methods, Jahresber Dtsch Math-Ver (2010) 112: 119-158

[18] G. N. Watson, A Treatise on the Theory of Bessel Functions, The University Press, Cambridge, 1944.

Zalman Balanov, Department of Mathematics, Xiangnan University, Chenzhou, Hunan 423000, China, and, Department of Mathematical Sciences, University of Texas at Dallas, Richardson, tX 75080, USA E-mail address: balanov@utallas.edu

Edward Hooton, Institute of Mathematics, Czech Academy of Sciences, Žitná 25, 11567 Praha 1 , Czech Republic

E-mail address: hooton@math.cas.cz

Wieslaw Krawcewicz, Applied Mathematics Center at Guangzhou University, Guangzhou 510006, China, and, Department of Mathematical Sciences, University of Texas at Dallas, Richardson, TX 75080, USA.

E-mail address: wieslaw@utallas.edu

Dmitri Rachinskit, Department of Mathematical Sciences, University of Texas at Dallas, RichardSON, TX 75080, USA

E-mail address: dmitry.rachinskiy@utdallas.edu 